\title{
Meeting report: stem cell biology and epigenetics
}

\author{
Joon-Kyu Lee $\cdot$ Keun Il Kim $\cdot$ Nam-Soo Kim
}

Received: 5 September 2013 / Accepted: 15 September 2013/Published online: 3 October 2013

(C) The Genetics Society of Korea 2013

The Genetics Society of Korea (GSK) had a symposium in the 68th Annual Meeting of the Korean Association of Biological Sciences (KABS) that was held during August 12-13 at Sogang University, Seoul, Korea. The symposium had two sessions on the two frontier fields in genetics; stem cell biology and epigenetics.

The first session was on the stem cell biology that covered from the basics of the stem cell research to progresses in therapeutic approaches. Three active scientists presented their recent findings. The first speaker was Professor Dong Wook Han at the Konkuk University, Korea, with a title of "Inducing different stem cell fates by defined factors". Because the audience consists of diverse fields in genetics, he began his talk with nice introduction on the definition and sources of the stem cells. Recent advances in stem cell research have revealed that cell-type specific transcription factors can rest the somatic memory of differentiated cells and induce direct reprogramming into specific cell identity. The most remarkable achievement is that a combination of neural-specific transcription factors can induce a neural stem cell (NSC) fate on the fibroblasts. The experimental results showed that the induced neural stem cells (iNSCs) were similar to the wild type NSCs in

\section{J.-K. Lee}

Department of Biology Education, Seoul National University, Seoul 152-742, Korea

\section{K. I. Kim}

Department of Biological Science, Sookmyung Women's University, Seoul 140-742, Korea

\section{N.-S. Kim $(\square)$}

Department of Molecular Bioscience, Institute of Bioscience and Biotechnology, Kangwon National University, Chuncheon 200-701, Korea

e-mail: kimnamsu@kangwon.ac.kr cell morphology, gene expression, epigenetic features, differentiation potential, and cellular function. Thus, he posited that cell type-specific defined factors can induce specific stem cell identities from somatic cells. He also introduced his recent findings on the direct conversion of differentiated somatic cells into specific cellular identities such as hepatocytes.

The second speaker professor, Hyuk-Jin Cha, at the Sogang University, Korea, gave a talk with a title of "Inhibition of pluripotent stem cell derived teratoma formation by small molecules". One of the obstacles in therapeutic application of the stem cells is the removal of residual undifferentiated pluripotent stem cells (PSCs) after differentiation. Because the undifferentiated PSCs can proliferate unlimitedly to form a tumor mass, it is critical to sort completely out the residual undifferentiated PSCs for tumor-free cell therapy. Several approaches have been suggested to eliminate the remaining undifferentiated cells, including the introduction of suicide gene, immunedepletion and introducing cytotoxic antibodies. However, the rigorous sorting techniques are still far behind in isolating the differentiated cells purely and have yet reached at the level of clinically viable strategy to eliminate teratoma formation. PSCs are highly susceptible to apoptotic stimuli such as reactive oxygen species and DNA damage stress which can induce genetic alteration to the differentiated cells. Thus, high responsiveness to apoptosis can be considered to be one of the protective mechanisms to retain genetic integrity during differentiation. Dr. Cha's group analyzed the pro- and anti-apoptotic genes in human PSCs (hPSCs) and found that 22 pro-apoptotic genes and 10 antiapoptotic genes were highly expressed in undifferentiated PSCs. Of interest, inhibition of two anti-apoptotic factors (BIRC5 and BCL10) by small molecule could significantly induce selective cell death of PSCs but not differentiated 
counterparts. In special, inhibition of BIRC5 gene product by quercetin (QC) or YM155 was able to selectively eliminate undifferentiated hPSCs with minimum effect on the differentiated cells such as dopaminergic neurons and smooth muscle cells and completely block teratoma formation. The selective cell death of hPSCs by QC or YM155 is closely associated to the mitochondrial apoptotic signal that was trigged by p53 mitochondrial localization. These results suggest that small molecules targeting antiapoptotic factors in undifferentiated hPSCs would be the clinically viable strategy to eliminate remaining undifferentiated hPSCs and to prevent teratoma formation for tumor-free hPSCs based cell therapy.

The third speaker was Dr. Byung-Rok Do, a CEO of the biotech venture firm, Hurim Biocells Inc., Korea with a title of "Preclinical trial for stem cell therapy". Stem cells have proven to have enormous potential in treatment of incurable diseases. However, it is highly necessary to introduce procedures of preclinical studies in stem cell therapy. To develop the cell therapy products, multi-step verification processes are required for safety testing before human trial. After thorough verification at the lab-basedlevel studies for efficacy and therapeutic potential of the cell therapeutic products, the in-house-level preclinical studies using disease model animals are followed, which determine therapeutic indications, efficacy and treatment procedures/methods. Because cells themselves are therapeutic products in cell therapeutics, it is very important to produce cells with safety. Thus, every manipulation of cells destined to human therapy has to be accomplished in a clean room equipped with specially designed work areas, which is under a constant control of air purity, personnel clothing, cleaning procedures, and so on. In general, cell therapy products have to be produced under the standard quality management protocols in every step, from collection to transportation, processing, testing, and release of cells, with credible efficacy controls. The produced cells have to be clearly proven their safety and efficacy from the accredited testing laboratories (GLP) outside in a proper way, as common in drug proceeds. The therapeutic efficacy of the cells must be tested in the proper disease animal model after transplantation. The processes have to be carried out in accordance with the laws of the Pharmaceutical Affairs Law, etc. Thus, during preclinical studies, information of cells' physical, chemical, and biological properties are created. These results of preclinical studies are basic documents for the application clinical trials to Korea Food and Drug Administration (KFDA). After reviewing the preclinical data, KFDA charters clinical trials. Clinical trials are also strictly processed according to the laws of the Pharmaceutical Affairs Law.

Before starting the second session, four young scientists gave talks briefly on their Ph.D thesis research. Dr.
Namhee Jeong, Chungbuk National University, talked about $L n$ gene which regulates leaf shape and number of seeds per pod in soybean with a title of "Ln is a key regulator of leaflet shape and number of seeds per pod in soybean". The Ln gene, homologous to Arabidopsis JAG$G E D(J A G)$ which regulates lateral organ development and has a pleiotropic effect on fruit ripening, was named as $G m-J A G 1$. Introducing the $G m-G A G 1$ to jagged mutant, Arabidopsis showed partial restoration of the phenotype in Arabidopsis. Because the Gm-GAG2, a homolog to $G m$ $G A G 1$, showed different expression in multiple tissues in soybean, he proposed that the $G m-G A G$ may have been sub- or neo-functionalized in soybean. The second speaker in young scientist session was Dr. Ja-Rang Lee at Pusan National University. She gave a talk with a title "Genome instability of retroelements by radiation treatment". Radiation induces genome instability via changes in genome structure, epigenetic regulation, and gene expression. Our genome carries various human endogenous retroviruses (HERVs). The expression of the HERVs is repressed by epigenetic mechanisms. qPCR showed that expression of HERVs was elevated in $\gamma$-irradiated human cells compared to the non-irradiated cells. She presented molecular evidences that epigenetic changes, especially chromatin modification, by irradiation are responsible for the HERV expression which induces the genome instability and ultimately pathogenesis. The third young scientist was JaeSung Lee, Hanyang University, Korea. His topic was "Whole genome data and its potential use in the molecular ecotoxicological research of the self-fertilizing fish, Kryptolebias marmoratus". Kryptolebias marmoratus is the only vertebrate species reproducing by self-fertilization. It has short generation time (3-4 months) and easy rearing under laboratory conditions. Another advantage is that $K$. marmoratus is highly resistance in extreme environmental conditions to render it to model species in toxicology study. In order to get genomic insights, whole genome sequencing was carried out on the $K$. marmoratus genome. Dr. Lee introduced the genome platform of the $K$. marmoratus and proposed adding the $K$. marmoratus to another model species for genomics in aquatic environmental toxicology. Professor Sun-Hee Lim, Donga University, Korea, delivered a talk on behalf of the last young scientist, Dr. Hyun-Hee Lee, with a topic of "Tristetraprolin regulates expression of ARE-containing genes and tumorigenesis in human cancer". Tristetraprolin (TTP) is an AU-rich element-binding protein that regulates mRNA stability. She reported that TTP suppresses the growth of human cancer cells both in vivo and in vitro by regulating the expression of vascular endothelial growth factor (VEGF) and LAST2. With several solid molecular evidences, they proposed that TTP acts as a negative regulator of VEGF and LAST2 gene expression in colon and lung 
cancer cells possibly by tumor- or sequence-specific pathway.

The last session was on the chromatin structure and epigenetics, where four eminent scientists presented excellent talks. The first speaker was Sang Kyu Lee, Kyungbook National University, Korea with a topic of "Systematic characterization of 67 histone marks and histone lysine crotonylation as a novel modification". A core histone protein typically consists of an unstructured $\mathrm{N}$-terminus, a globular core including a central histone-fold domain, and conformationally mobile C-terminal tail. Professor Lee's group identified 67 previously undescribed histone modifications. The unique structure and genomic localization of histone lysine crotonylation (Kcr) suggested that it is mechanistically and functionally different from the histone lysine acetylation (Kac). The histone Kcr marks either active promoters or potential enhancers in human somatic and mouse germ cells. Lee's group investigated the Kcr marks during spermatogenesis in which Kcr is enriched in sex chromosomes and specifically marks testisspecific genes, including a significant proportion of $\mathrm{X}$-linked genes that escape the chromosome inactivation in haploid cells, immediately after meiosis. These results suggested dramatic extension of the repertoire of post translational modification sites and Kcr marks specific to the active sex chromosome linked genes in post-meiotic male germ cells.

The second speaker was professor Jong-Soo Lee at Ajou University, Korea. Her topic was "Epigenetic regulation in response to DNA damage". Both genetic and epigenetic mechanisms induce genome instabilities that have often leads to tumorigenesis and tumor progression. In response to the DNA damage, chromosome structures are dynamically regulated spatially and timely. Professor Lee gave a talk on the heterochromatin protein $1 \gamma(\mathrm{HP} 1 \gamma)$, a chromatin protein, regulates BRCA1-mediated DNA repair and transcription via modulation of damaged DNA or promoter occupancy and histone modification. Mammalian heterochromatin proteins 1 (HP1 $\alpha, \mathrm{HP} 1 \beta, \mathrm{HP} 1 \gamma)$ are non-histone proteins that interact with a set of proteins that play a role in chromosome silencing, transcription, remodeling, and generating pleiotropic effects. She demonstrated that HP1 $\gamma$ interacts with BRCA1 through its chromoshadow domain. BRCA1 interacts with wild type HP1 $\gamma$ and a mutant deficient in methylated-histone (H3K9me) binding (HP1 $\gamma$ V22A). HP1 $\gamma$ and HP1 $\gamma$ V22A suppress BRCA1-mediated transcription by causing BRCA1 to be disassembled from the BRCA1 target promoter and augmenting $\mathrm{H} 3 \mathrm{~K} 9 \mathrm{me}$. By contrast, another HP1 $\gamma$ mutant deficient in binding to PXVXL/I motif (HP1 $\gamma \mathrm{W} 164 \mathrm{~A})$ fails to interact with BRCA1 and to repress transcription. Consistently, HP1 $\gamma$ and HP1 $\gamma$ V22A associate with the promoter in SUV39H1knockdown cells, suggesting that HP1 $\gamma$ binds to BRCA1- traget promoter and represses its transcription in the BRCA1-HP1 $\gamma$ interaction dependent manner, accompanied by BRCA1 disassembly. Thus, they searched for temporal differences between BRCA1-HP1 $\gamma$ assembly/disassembly at the promoter. The time-lapse studies on promoter association and histone-methylation following DNA-damaging, reveal that BRCA1 is initially assembled at the promoter, $\mathrm{HP} 1 \gamma$ in turn recruited, and finally SUV39H1 is targeted there. Following the HP1 $\gamma / \mathrm{SUV} 39 \mathrm{H} 1$ assembly on the promoter, the resultant disassembly of BRCA1 and histonemethylation leads to repression. Together, she proposed that HP1 $\gamma$, through interaction with BRCA1, is guided at the BRCA-target promoter, and HP1 $\gamma$ functions in the activation-repression switch of BRCA1-mediated transcription.

The third speaker was Sang-Beom Seo, Chung-Ang University, Korea, with a title of "Histone methylation and demethylation mediated transcriptional regulation in leukemia". Histone lysine methylation and demethylation are considered critical steps in transcriptional regulation. Using chromatin immunoprecipitation with microarray technology (ChIP-chip) analysis to examine the genome-wide occupancy of H3K9-me2 during all-trans-retinoic acid (ATRA)-induced differentiation of HL-60 promyelocytic leukemia cells, his group found that KDM3B, which contains a JmjC domain, was down-regulated during differentiation through the recruitment of a co-repressor complex. They verified that KDM3B expression was downregulated and that the $\mathrm{KDM} 3 \mathrm{~B}$ promoter was enriched with H3K9-me2 during ATRA-mediated differentiation of HL60 cells. KDM3B demethylated specifically H3K9-me2 in vivo and in vitro. KDM3B induced leukemogenic oncogene lmo2expression via a synergistic interaction with CBP. A total of 112 genes were either up- or down-regulated by more than 1.5-fold when KDM3B was depleted. Notably, among the genes regulated by KDM3B, lmo2 was found to be down-regulated in the microarray analysis when KDM3B was depleted. ChIP-qPCR data suggest that KDM3B specifically targets the $1 m o 2$ promoter and activates transcription through $\mathrm{H} 3 \mathrm{~K} 9-\mathrm{me} 1 / 2$ demethylase activity. Thus, he suggested that KDM3B and the CBP HAT complex are both physically and functionally associated in transcriptional activation of $1 m o 2$. FACS analysis results led them to conclude that regulation of $1 m o 2$ by KDM3B is crucial for HL-60 cell differentiation. Moreover, their results showed that KDM3B repressed leukemia cell differentiation and was up-regulated in blood cells from acute lymphoblastic leukemia (ALL)-type leukemia patients. The combined results of this study provide evidence that the H3K9-me1/2 demethylase KDM3B might play a role in leukemogenesis via activation of $1 \mathrm{mo} 2$ through interdependent actions with the histone acetyltransferase (HAT) complex containing CBP. Using ChIPchip analysis, they also found that the H3K9-me2 target 
gene $J A K 2$ was an important factor during differentiation of the HL-60 promyelocytic leukemia cell line by ATRA treatment. The JAK2 is located on human chromosome 9 (9p24), and the H3K9-me2 target site is located in the $J A K 2$ promoter, indicating modification during differentiation. Thus, he suggested that increased H3K9 HMTase G9a represses JAK2 expression specifically in a SET domain-dependent manner during ATRA-mediated HL-60 differentiation. They found that G9a knockdown repressed ATRA-mediated HL-60 cell differentiation, suggesting that G9a interacts with YY1 in vivo and mediates transcriptional repression of $J A K 2$ in an YY1-dependent manner. They also demonstrated that the repression of JAK2 transcription results in the down-regulation of H3Y41 phosphorylation on the $1 m o 2$ promoter. Thus, G9a induces cell differentiation via negative regulation of $J A K 2$ transcription. Repression of $J A K 2$ transcription by G9a decreased H3Y41 phosphorylation and promoted inhibition of the recently identified JAK2-H3Y41P-HP1 $\alpha$ pathway-mediated leukemogenesis.

The last speaker was Professor AeRi Kim, Pusan National University, Korea, with a title of "Histone acetylation contributes to chromatin looping between the locus control region and globin gene by influencing hypersensitive site formation". Transcription regulatory elements are generally located in long distances on the eukaryote genome, but often positioned in close proximity in the threedimensional space of nucleus, forming chromatin loops. Although the regulatory elements have highly acetylated histones, it is unclear whether histone acetylation affects chromatin loop formation and how the acetylation contributes to the loop formation. In the mammalian $\beta$-globin locus, the locus control region (LCR), a far upstream enhancer, is closely positioned to the transcriptionally active globin genes in erythroid cells. Histones are highly acetylated in the LCR and active globin genes. In order to understand the relationship of histone acetylation to chromatin loop formation, Professor Kim's group inhibited histone acetylation of the $\beta$-globin locus by reducing histone acetyltransferases CBP and p300 in K562 cells and examined the chromatin structure including chromatin loops, histone modifications, hypersensitive site (HS) formation and activator binding in the $\beta$-globin locus. Histone hypoacetylation in the $\beta$-globin LCR decreased transcription of the human $\gamma$-globin genes. The proximity between the LCR and the active $\gamma$-globin gene was decreased in the hypoacetylated $\beta$-globin locus. Loss of acetylation at histone H3K27 increases mono- and di-methylation in the LCR and $\gamma$-globin genes. Sensitivity to DNase I attack and binding of erythroid specific activators were reduced in the hypoacetylated LCR hypersensitive sites (HSs) and gene promoter. Interestingly, the chromatin loop between insulators, HS5 and $3^{\prime} \mathrm{HS} 1$, was formed regardless of the hypoacetylation of the $\beta$-globin locus. CTCF binding was maintained at HS5 and $3^{\prime} \mathrm{HS} 1$ in the hypoacetylated locus. These results indicate that histone acetylation play a role in chromatin loop formation by generating hypersensitive chromatin structure in the LCR/enhancer and promoter, which might allow the binding of activators and co-activators mediating chromatin loop between them. When the chromatin loop is finally formed, the gene will be transcribed. However, chromatin looping between insulators appears to be independent from histone acetylation, which might be established in a different manner from loops between the LCR and promoter. 